\title{
ATM Adaptation Layer Issues
}

Arne A. Nilsson

Zhi Cui

Center for Communications and Signal Processing Department of Electrical and Computer Engineering North Carolina State University

\author{
TR-92/22 \\ November 1992
}




\title{
ATM Adaptation Layer Issues
}

\author{
Arne A. Nilsson and Zhi Cui \\ Center for Communications and Signal Processing \\ Dept. of Electrical and Computer Engineering \\ North Carolina State University \\ Raleigh, N.C. 27695-7914
}

\begin{abstract}
The impact of the Convergence Sublayer Protocol Data Unit (CS-PDU) size on the end-to-end network delay in a $B-I S D N$ environment is investigated in this paper. Cell loss probabilities are assumed to be bursty and when a cell loss occurs a retransmission on the $C S-P D U$ level will be done. The network has been simulated and the simulation results indicate that it is possible to select a CS-PDU size that will minimize the end-to-end delay. A typical ATM network has also been analyzed by using mathematical modeling technique. Assuming Poisson arrival process, it is possible to compute approximately the end-to-end delay as a function of the CS-PDU size. It can then be shown that the delay is optimized for a particular CS-PDU size.
\end{abstract}

\section{Introduction}

The Asynchronous Transfer Mode (ATM) is a fast packet switching and multiplexing technique for broadband ISDN. In an ATM network, all information ranging from narrowband voice and data traffic to broadband video traffic is transmitted using a fixed size "cell". In an ATM network, see Figure 1, above the ATM layer is an adaptation layer (AAL) which is used to support the connection between ATM and non-ATM interfaces [1] [2]. The AAL isolates the higher layers from the specific characteristics of the ATM layer by mapping the higher layer Protocol Data Units(PDUs) into the information field of the ATM cell and vice versa. It is likely that packet sizes at the transport level will be fairly large (64KBytes to a few Megabytes). Since ATM cells only have a payload of 48 bytes or 44 bytes, this implies a segmentation and reassembly process involving potentially tens of thousands of cells per packet. For a trans-

\begin{tabular}{|c|}
\hline Higher Layers \\
\hline AAL \\
\hline ATM \\
\hline Physical \\
\hline
\end{tabular}

Figure 1: ATM Network Layers.

port layer protocol, without error correction capabilities which retransmits errored USER-PDUs, significant operational issues arise such as: how to deal with lost cells, how to properly reassemble a packet from cells in a multipoint environment, how to reduce the system end-to-end delay, and how to increase system throughput for large file transfer [3].

In this paper, we propose an intermediate segmentation techniques as a method to break up large transport layer packets into several sub-packets to reduce end-to-end delay and increase data transfer throughput in the face of bit errors and ATM cell loss. An important design issue is to determine the optimal sub-packet size. In section 2 , we describe the system and queueing model in detail and present simulation results for the optimal sub-packet size. In Section 3, we present the exact analysis of the end-to-end delay for a Poisson arrival system.

\section{Model description}

In this section, we describe in detail the CS-PDU and network architecture, cell-error probability and 


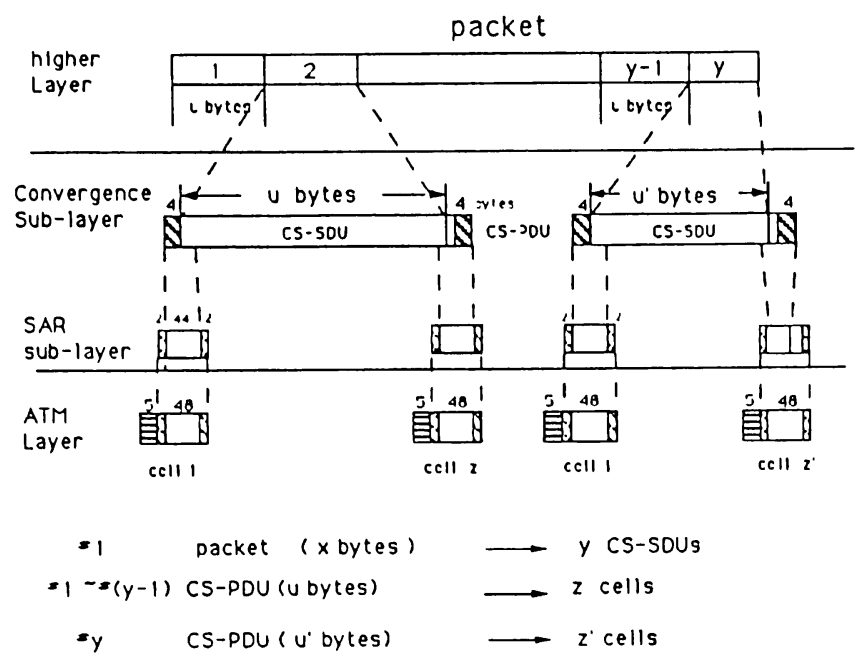

Figure 2: CS-PDU Information.

simulation result for the end-to-end delay as a function of CS-PDU size.

\section{$2.1 \quad$ CS-PDU}

The AAL consists of two sub-layers: Convergence Sub-layer(CS) and Segmentation And Re-assembly Sublayer(SAR) [4]. see Figure 2. A USER-PDU that enters the CS layer is divided up into several subpackets so called CS-SDU:s. After processing the CS layer, adds header and trailer to the CS-SDU and thus a CS-PDU is formed. At the receiving side of the network, these CS-PDUs will be reassembled to packets. In the SAR-sublayer, we segment the CS-PDU into a size suitable for the information field of an ATM cell, and reassemble the contents of ATM cell information fields into CS information.

\subsection{Queueing Network Architecture.}

Based upon the AAL functions, the queueing network structure, as shown in Figure 3 , can be derived. At the source, we use queues split1 and split2 to simulate the functions of the CS and SAR-sublayers, respectively. Cells are transmitted into the ATMnetwork which is assumed to have finite capacities and low cell loss probability. At the destination, the cells are reassembled to CS-PDU and packets. If an error happens either by cell loss or by bit error, the particular CS-PDU will be retransmitted.

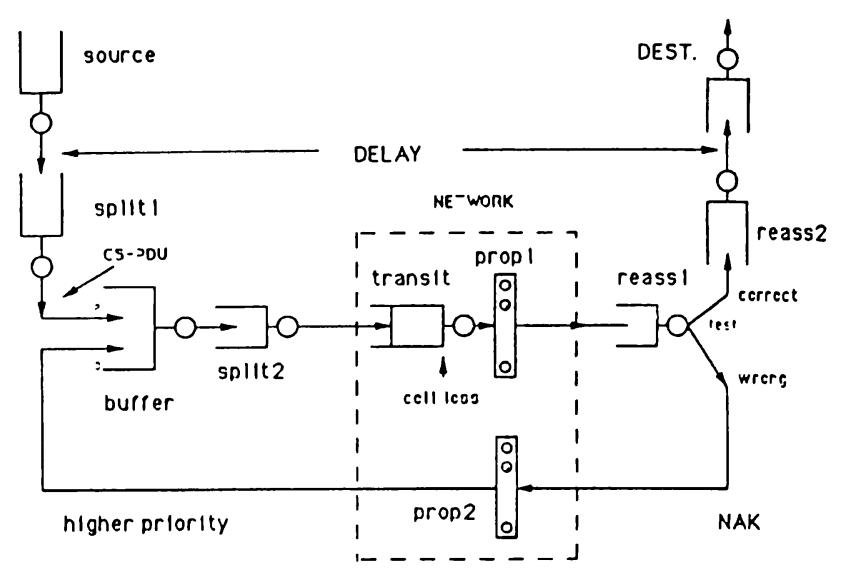

Figure 3: ATM Network Architecture.

\subsection{Error Probability}

In high speed networks, fiber optics is often the selected transmission medium. As a matter of fact, standards imply that the Synchronous Optical NETwork (SONET) is the preferred architecture. It is well known that in a fiber optic based network, the probability of bit error is very low, typically less than $10^{-10}$. Thus it is not likely that an ATM cell will be corrupted due to a bit error. It is much more likely that an ATM cell will be lost or destroyed due to buffer overflow or a lack of some network resource. In a lightly loaded network the chance of an ATM cell loss is very low of course. There is, however, a chance of loss during those periods of time when the network load is temporarily high. Note that this happens even in lightly loaded networks. We have adopted as a model for the probability of cell loss a simple two state model. In one of the states the cell loss probability is very small, typically negligible, and this happens when the amount of information in the network is smaller than the network system capacity. In the other state the cell loss probability is typically high and this, of course, happens when the amount of information in the network is significant, see Fig. 4.

\subsection{Simulation}

Given a cell loss probability behavior as presented in Figure 4, we simulate the queueing network [see Figure 3] to find the mean end-to-end delay and maximum end-to-end delay in terms of the CS-PDU size. The simulation results are presented in Figure 5 and 


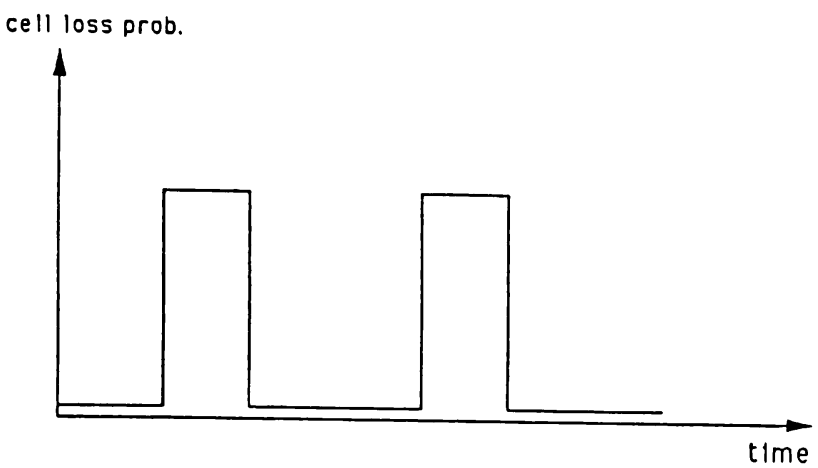

ligh cell loss Prob. low cell loss Prob.

when Info. bigger than syster capacity when info. sir aller than syster capacity

Figure 4: Cell loss probability

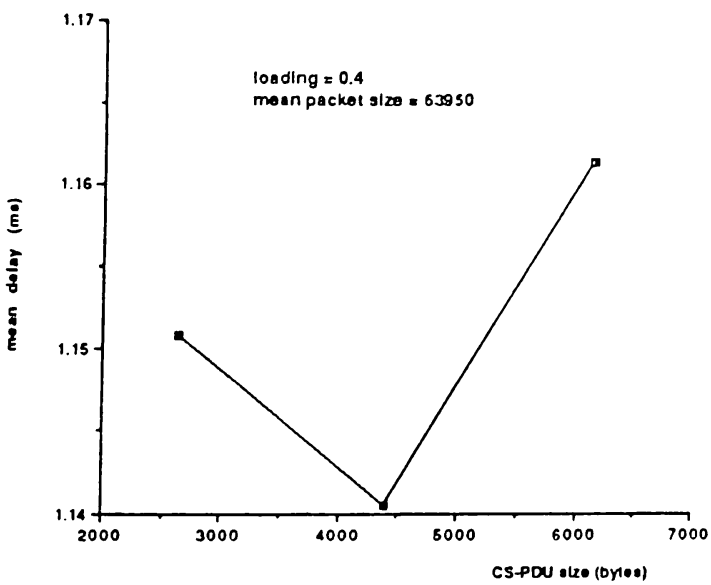

Figure 5: Mean end-to-end delay

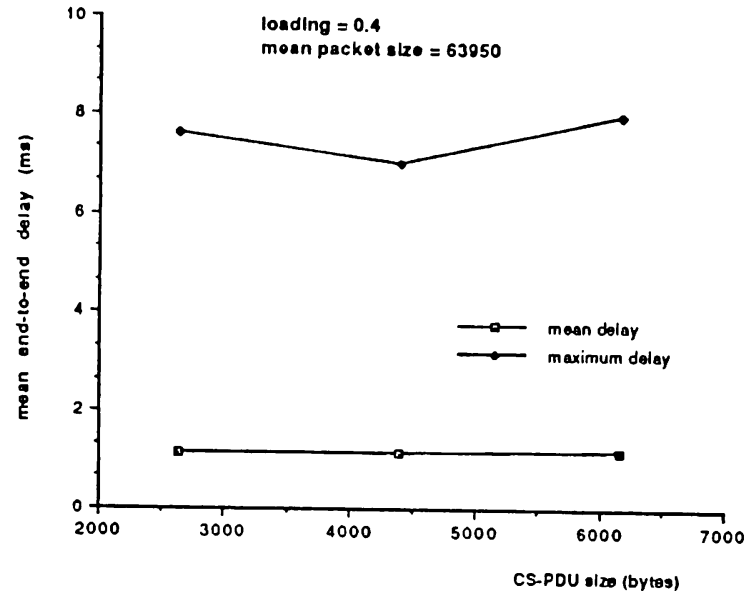

Figure 6: Maximum end-to-end delay and mean endto-end delay

Figure 6, respectively. From the simulation results, we can conclude:

1) the mean end-to-end delay depends upon the error probability distribution and CS-PDU size;

2) an optimal CS-PDU size under a given cell loss distribution can be found.

3) CS-PDU size has much more influence on the maximum end-to-end delay than on the mean end-to-end delay;

\section{Analytical result}

Our first attempt to analyse the system assumes naively a Poisson process as the packet arrival process to the CS layer. This implies that we can simplify our network [Fig 3] into the queueing network as shown in Figure 7.

Here $\lambda$ is the packet arrival rate; $\mathrm{X}$ is the mean big packet size measured in cells; $\frac{1}{\mu}$ (cells)is the CS-PDU size; $\mathrm{X} \mu$ is the number of cells in a mean size packet; $\mathrm{P}$ is the probability of retransmitting a CS-PDU, $\mathrm{P}=\frac{e}{\mu}$; where $\varepsilon$ is the mean cell loss probability; $\mu_{p}$ is the service rate for the propagation delay in finite server and D is the delay for a CS-PDU. For open network [Fig.7], according to Little's result [5] 


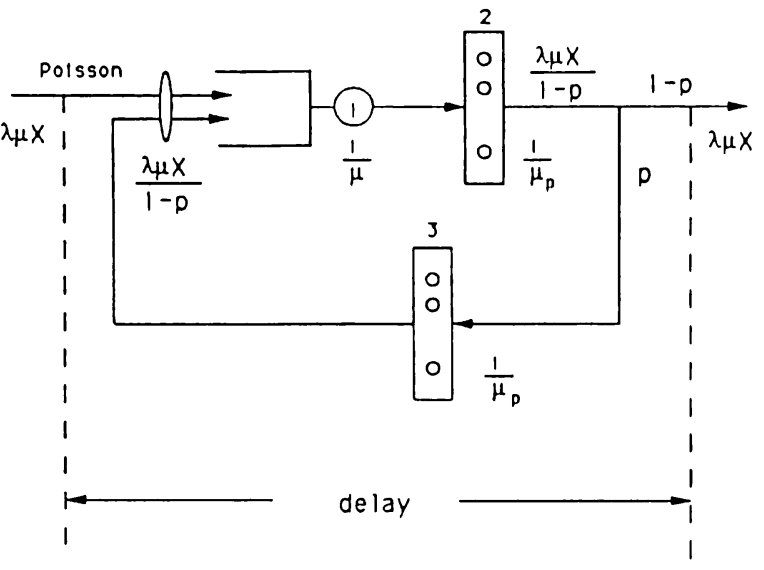

Figure 7: Analytical Queueing Network Model

$$
\begin{aligned}
D & =\frac{\text { average number of } C S-P D U_{s} \text { in network }}{\lambda} \\
& =\frac{N 1+N 2+N 3}{\lambda}
\end{aligned}
$$

where $N_{i}$ is the average number of CS-PDUs in station $i$. For the $M / M / 1$ queue 1 ,

$$
\begin{aligned}
N_{1} & =\frac{\rho_{1}}{1-\rho_{1}} \\
& =\frac{\lambda \mu X}{(1-P) \mu-\lambda \mu X} .
\end{aligned}
$$

For the $M / M / \infty$ queues 2 and 3 , we find

$$
\begin{aligned}
N_{2} & =\rho_{2} \\
& =\frac{\lambda \mu X}{(1-P) \mu_{p}} . \\
N_{3} & =\rho_{3} \\
& =\frac{\lambda \mu X P}{(1-P) \mu_{p}} .
\end{aligned}
$$

Trivial computations give us

$$
D=\frac{1}{(1-p) \mu-\lambda \mu X}+\frac{1+p}{(1-p) \mu_{p}} .
$$

Figure 8 shows the exact analysis result for the mean end-to-end delay as a function of CS-PDU size.

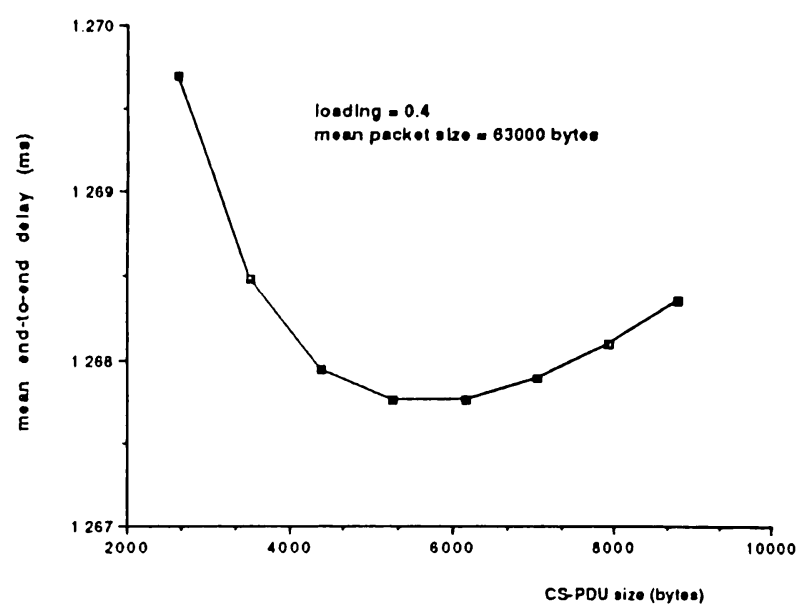

Figure 8: Analytical Result for end-to-end delay

\section{Conclusion}

In this paper, we have presented results obtained from a simulation program that permits us to demonstrate the effect of how big packets should be broken up into smaller packets (sub-packets) before being fragmented into ATM cells. The simulation program permits us to vary the cell loss probability as a function of buffer size. Given a cell loss distribution, we can use the simulation program to find the optimal sub-packet size. For a Poisson arrival system, we also obtained the exact analytical result for the end-to-end delay.

\section{References}

[1] S.E. Minzer, "Broadband ISDN and Asynchronous Transfer Mode (ATM)", IEEE Communications Magazine, vol.27, no.9, 1989.

[2] M. J. Rider, "Protoculs for ATM Access Networks", GLOBECOM, vol.1, 1988.

[3] "VISTAnet: A Very Iligh Bandwidth Prototype Network for Interactive 3D Medical Imaging ", Research Plan by Bellsouth Services, GTE Corp., MCNC, UNC-Chapel Hill, 1990.

[4] CCITT Recommendations Drafted by Working Party XVIII/8, no.6, 1988

[5] L. Kleinrock, Queueing System, Vol. I, John Wiley \& Sons, Inc, New York, NY, pp.134-136, 1975. 